\title{
A single dose of dezocine suppresses emergence agitation in preschool children anesthetized with sevoflurane-remifentanil

Li-Jun An ${ }^{1,2}$, Yang Zhang ${ }^{2}$, Zheng Su², Xian-Long Zhang ${ }^{2}$, Hai-Lin Liu², Zhi-Jie Zhang ${ }^{2}$, Jian-Lin Hu' and Shi-Tong Li ${ }^{1 *}$ (D)

\begin{abstract}
Background: Emergence agitation (EA) is a common phenomenon in preschool children during emergence from general anesthesia. This study evaluated the safety and efficacy of dezocine for emergence agitation in preschool children anesthetized with sevoflurane-remifentanil.

Methods: A total of 100 preschool children, scheduled for elective laparoscopic repair of an inguinal hernia by high ligation of the hernia sac under sevoflurane-remifentanil anesthesia were randomized into two groups: Group $C(n=50)$ received Ringer's lactate $10 \mathrm{~mL}$ and Group D received Ringer's lactate $10 \mathrm{~mL}$ containing dezocine $0.1 \mathrm{mg} / \mathrm{kg}$, postoperatively.

Results: Incidence of EA, defined as a score $\geq 3$ on Aono's four point scale or Pediatric Anesthesia Emergence Delirium (PAED) score $\geq 10$ in the PACU (10\% vs. 76\%) and the percentage of patients with severe EA (PAED score $\geq$ 13) $(12 \%$ vs. $76 \%)$ were significantly lower in Group $D$ compared to Group $C(P<0.05)$. Mean Children and Infants Postoperative Pain Scale (CHIPPS) score was significantly lower in Group D compared to Group C (1.2 \pm 0.5 vs. $5.2 \pm 0.6$; $P<0.05)$. Patients need for fentanyl ( $18 \%$ vs. $4 \%$ ) or propofol rescue ( $20 \%$ vs. 0 ) was significantly greater in Group C compared to Group D. No significant differences in other relative aspects after surgery between groups.

Conclusion: Administration of dezocine $0.1 \mathrm{mg} / \mathrm{kg}$ decreased the incidence and severity of EA in preschool children that had undergone laparoscopic repair of an inguinal hernia by high ligation of the hernia sac under sevofluraneremifentanil anesthesia.
\end{abstract}

Trial registration: A single dose of dezocine suppresses emergence agitation in preschool children anesthetized with sevoflurane-remifentanil effectively: A double-blind, prospective, randomized, controlled study, Chinese Clinical Trial Registry (ID: ChiCTR-IOR-16010033), retrospectively registered on November 21, 2016.

Keywords: Dezocine, Emergence agitation, Preschool children, Sevoflurane-remifentanil

\section{Background}

Laparoscopic repair of an inguinal hernia by high ligation of the hernia sac is a common minimally invasive pediatric surgical procedure. Sevoflurane, combined with low-dose remifentanil, is an anesthetic procedure that has been widely used in hernia surgery. Although sevoflurane-remifentanil anesthesia is associated with a smooth onset, rapid induction, prompt emergence, and

\footnotetext{
*Correspondence: shlishitong@126.com

'Department of Anesthesiology, Shanghai General Hospital of Nanjing Medical University, University, No. 100 Haining Road, Shanghai 200030, China Full list of author information is available at the end of the article
}

fast recovery, it can result in emergence agitation (EA) [1]. EA is defined as psychomotor agitation during emergence from general anesthesia, and is a frequent problem among pediatric patients [2]. Indeed, the incidence of EA has been estimated at $10-66 \%$ in pediatric patients following sevoflurane-remifentanil anesthesia [3].

EA can result in physical harm to the child or to surgical staff. Although self-limiting, EA is disturbing for both parents and care-givers [4]. Evidence suggests that causative factors of EA in preschool-aged children include rapid emergence, psychological and neurological immaturity, 
sevoflurane-induced noradrenaline release in the brain, pain, and anxiety [5].

As pain and anxiety are implicated in the etiology of $\mathrm{EA}$, analgesic and sedative agents such as midazolam, $\alpha 2$ adrenergic receptor agonists such as propofol, and opioids have been used to minimize EA [6-10]. These therapies have various degrees of success, as well as their own respective side effects when treating EA [11].

Dezocine is an analgesic and sedative that is used for perioperative pain management. We hypothesized that postoperative dezocine administration may decrease the incidence and severity of EA in pediatric patients. Dezocine is a full agonist of the $\mathrm{K}$-receptor and partial agonist of the $\mu$-receptor without $\mu$-receptor dependence. The analgesic effect of dezocine is approximately equipotent with morphine. It has a minor ceiling effect for respiratory depression accompanying its analgesic activity, and its sedative effect is well tolerated in a single dose [12]. The objective of this study was to investigate the safety and efficacy of postoperative dezocine administration on the incidence and severity of EA in preschool children undergoing laparoscopic repair of an inguinal hernia by high ligation of the hernia sac under sevofluraneremifentanil anesthesia.

\section{Methods}

\section{Study population and study design}

Preschool children undergoing laparoscopic repair of an inguinal hernia by high ligation of the hernia sac at our institution between December 2014 and November 2016 were eligible for this study. Inclusion criteria were 1) American Society of Anesthesiologists (ASA) score I or II; and 2) scheduled for elective laparoscopic repair of an inguinal hernia by high ligation of the hernia sac under sevoflurane-remifentanil anesthesia. Exclusion criteria were 1) psychiatric or neurological disease; 2) sleep apnea; 3) treatment with sedative or opioid two weeks prior to surgery and 4) body mass index (BMI) $\geq 40 \mathrm{~kg} / \mathrm{m}^{2}$. Study participants were randomly allocated into Group C (control group, $n=50$ ) and Group D (dezocine group, $n=50$ ) using a computer generated table of random numbers.

This study was approved by the Ethics Committee of Huai'an First People's Hospital, affiliated with Nanjing Medical University, China (Approval \# HA141001S). Written informed consent was obtained from the patients' parents or legal guardians.

Children were fasted for $\geq 4 \mathrm{~h}$ before induction of anesthesia and received no premedication. Upon arrival in the operating room, heart rate (HR), pulse oximetry $(\mathrm{SpO} 2)$ and non-invasive blood pressure (BP) monitoring were applied. One experienced anesthesiologist administered anesthesia, performed tracheal intubation, and assessed intubation. Anesthesia was induced with
$8 \%$ sevoflurane in oxygen flowing at $6 \mathrm{~L} / \mathrm{min}$ through a pediatric anesthesia breathing circuit with a $1.5 \mathrm{~L}$ rebreathable bag. Initially, spontaneous respiration was assisted; subsequently, respiration was manually controlled. An intravenous cannula was sited after disappearance of the eyelash reflex. Dexamethasone $0.2 \mathrm{mg} / \mathrm{kg}$ and ondansetron $0.15 \mathrm{mg} / \mathrm{kg}$ were administered through the cannula. The concentration of sevoflurane was decreased to 1.3 minimum alveolar concentration (MAC). Tracheal intubation was attempted using a tracheal tube (size formula: internal diameter $=$ $4+$ yr./4), the muscle relaxant atracurium $1.0 \mathrm{mg} / \mathrm{kg}$, and fentanyl $1 \mu \mathrm{g} / \mathrm{kg}$. Anesthesia was maintained with 0.8-1.2 MAC sevoflurane in combination with remifentanil $(0.5 \mu \mathrm{g} / \mathrm{kg} / \mathrm{h}), \mathrm{BP}$ and HR within $20 \%$ of the baseline value, and bispectral index (BIS) values between 40 and 60. Ventilation was controlled to maintain end tidal carbon dioxide (ETCO2) levels between 35 and $40 \mathrm{mmHg}$ and oxygen flow at $2 \mathrm{~L} / \mathrm{min}$.

Skilled surgeons performed the operations. All incisions were infiltrated with $0.25 \%$ ropivacaine before surgery. At the completion of the surgery, remifentanil was discontinued and fentanyl $1 \mu \mathrm{g} / \mathrm{kg}$ was administered. Atropine $0.01 \mathrm{mg} / \mathrm{kg}$ and neostigmine $0.04 \mathrm{mg} / \mathrm{kg}$ were used to reverse residual neuromuscular blockade. Patients in Group $\mathrm{C}$ received $10 \mathrm{~mL}$ Ringer's lactate; patients in Group D received dezocine $0.1 \mathrm{mg} / \mathrm{kg}$ (Yangzi River Co., Jiangsu, China) in $10 \mathrm{~mL}$ Ringer's lactate. Concurrently, sevoflurane was terminated and controlled ventilation with $8 \mathrm{~L} / \mathrm{min}$ of oxygen was instituted. Extubation was performed when the cough reflex and regular respiration were regained. After extubation, subjects were transferred to the postanesthesia care unit (PACU). Oxygen $(6 \mathrm{~L} / \mathrm{min})$ was administrated via a face-mask in the PACU.

\section{Clinical evaluation}

Primary outcomes of the study were the incidence and severity of EA in the PACU. Secondary outcomes of the study were time to extubation, emergence time, Children and Infants Postoperative Pain Scale (CHIPPS) score, incidence of rescue drug administration, time in the PACU, and Ramsay sedation score.

Emergence time after extubation was defined as time to eye opening or purposeful movement in the PACU. Consciousness after emergence was evaluated using the Ramsay sedation scale [13]. In the PACU, a study investigator blinded to the study groups simultaneously assessed the incidence of postoperative EA defined as a score $\geq 3$ on Aono's four point scale or Pediatric Anesthesia Emergence Delirium (PAED) PAED score $\geq$ 10, and the severity of postoperative EA was described by the distribution of EA scores on the PAEDscale $[1,14]$; a score $\geq 13$ was classified as severe EA and a score $\geq$ 10 - <13 was classified as moderate EA. Postoperative 
pain was assessed by the CHIPPS [15]. A bolus of fentanyl $1 \mu \mathrm{g} / \mathrm{kg}$ was injected as a rescue medication for severe EA or a CHIPPS pain score $>5$. Propofol $1 \mathrm{mg} / \mathrm{kg}$ was administered if EA was not attenuated within $5 \mathrm{~min}$. Patients were discharged from the PACU to the ward when they recovered from anesthesia, defined as an Aldrete score $<9$ [16].

\section{Statistical analysis}

Statistical analyses were conducted using IBM SPSS Statistics for Windows Version 20.0 (IBM Corp., Armonk, NY, USA). Based on a previous study in children undergoing cleft lip surgery under sevoflurane anesthesia that reported a $25 \%$ decrease in the incidence of EA as clinically significant [17], the sample size for the current study was calculated as 50 patients in each group (alpha value, 0.05 ; beta-value, 0.80 ).

Continuous variables were analyzed with Student's $t$-test. Categorical variables including gender, ASA, and incidence of EA are reported as frequency, percentage, and risk ratios (RRs) with corresponding 95\% confidence interval (CIs) and were compared using chi-square test and correction or Fisher's exact probability test. A $P$ value less than 0.05 was considered statistically significant.

\section{Results}

\section{Study sample}

This study included a consecutive sample of 100 pediatric patients (55 male/23 female; mean age $4.6 \pm$
1.9 years, range $1-6$ years) (Fig. 1). All surgeries were successful. Two cases were lost to follow-up, one in Group $\mathrm{C}$ that experienced serious laryngospasm after extubation requiring the use of a muscle relaxant and one in Group D who suffered from bilateral inguinal hernia. The final analysis included 49 patients in Group C (29 male/20 female; mean age $4.9 \pm 1.6$ years, range 1-6 years) and 49 patients in Group D (26 male/23 female, mean age 4. $2 \pm 2.1$ years, range, 1-6 years). Patients' baseline demographic and clinical characteristics are shown in Table 1. There were no significant differences between groups.

\section{Outcomes}

Primary and secondary outcome data are shown in Table 2 and Fig. 2. Incidence of EA in the PACU $(10 \%$ vs. $76 \%$ and $27 \%$ vs. $86 \%, P<0.05)$, the percentage of patients with severe EA ( $88 \%$ vs. $46 \%, P<0.05)$, and the percentage of patients with moderate EA ( $12 \%$ vs. $54 \%$, $P<0.05)$ were significantly lower in Group D compared to Group C. There were no significant differences in mean time to extubation and mean emergence time between groups. Mean CHIPPS score was significantly lower in Group D compared to Group C (1.2 \pm 0.5 vs. $5.2 \pm 0.6 ; P<0.05)$. Patients need for fentanyl $(18 \%$ vs. $4 \%)$ or propofol rescue $(20 \%$ vs. $0 \%)$ was significantly greater in Group C compared to Group D. There was no significant difference in mean time to discharge from the PACU between groups. There were no significant differences in the incidence of postoperative events including

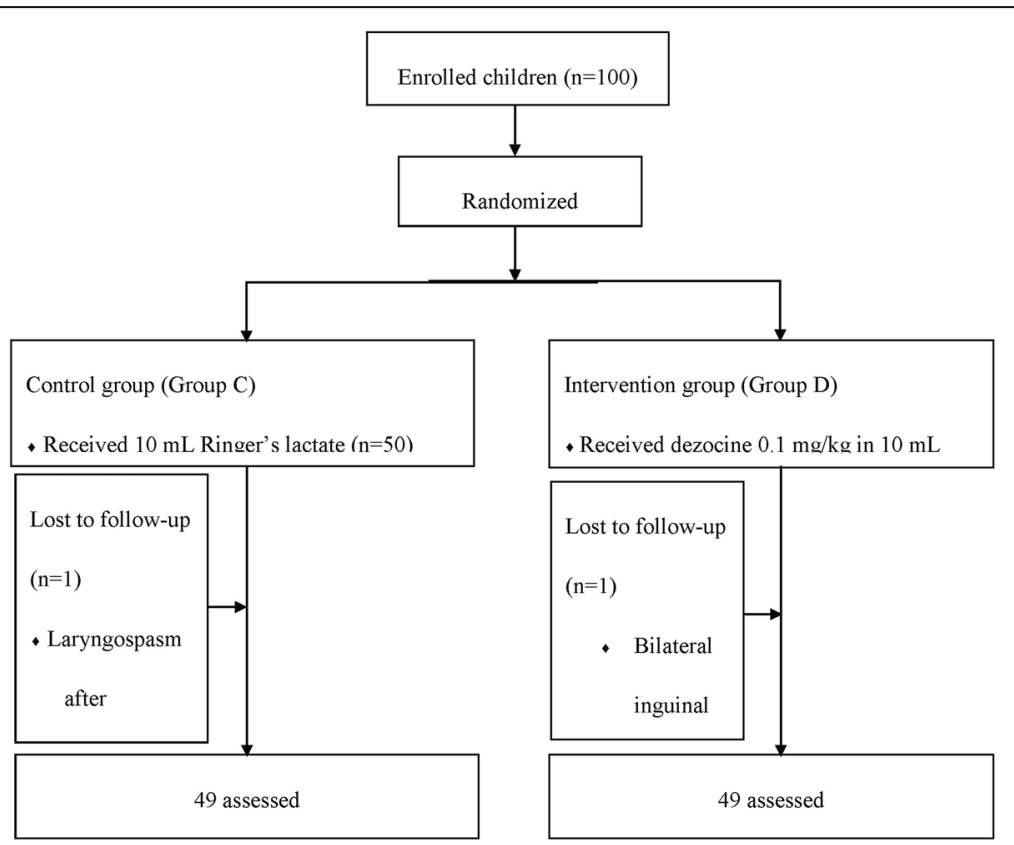

Fig. 1 CONSORT diagram. A total of 100 children were enrolled the study and allocated into two equal groups. Two children were lost to follow up, one because of bilateral inguinal hernia, the other due to laryngospasm after extubation and the use of muscle relaxant 
Table 1 Patients baseline demographic and clinical characteristics

\begin{tabular}{llll}
\hline & Group C $(n=49)$ & Group D $(n=49)$ & $p$-value \\
\hline Age $(\mathrm{yr})$ & $4.9 \pm 1.6$ & $4.2 \pm 2.1$ & 0.067 \\
Sex $(\mathrm{M} / \mathrm{F})$ & $29 / 20$ & $26 / 23$ & 0.541 \\
Weight $(\mathrm{kg})$ & $18.4 \pm 3.1$ & $17.5 \pm 4.3$ & 0.238 \\
Height $(\mathrm{cm})$ & $78.3 \pm 10.2$ & $80.5 \pm 12.3$ & 0.338 \\
BMI & $19.5 \pm 3.2$ & $20.1 \pm 2.8$ & 0.326 \\
ASA (I/II) & $46 / 3$ & $46 / 3$ & 1.000 \\
Duration of surgery (min) & $35.1 \pm 6.9$ & $36.8 \pm 8.0$ & 0.263
\end{tabular}

Data are presented as numbers or mean \pm SD. Group $C=$ Ringer's lactate, Group $D=$ dezocine $0.1 \mathrm{mg} / \mathrm{kg}$. M: male, F: female. BMI: Body Mass Index; ASA: American Society of Anesthesiologist

desaturation, headache, nausea, and vomiting between the two groups.

There was no significant difference in mean Ramsey sedation score between groups. However, more patients in Group $\mathrm{C}$ were evaluated as level 1, which indicates they were anxious, agitated or restless, compared to Group D, while more patients in Group D were evaluated as level 2 (cooperative, orientated, tranquil) or level 3 (responsive to commands only) compared to Group C (Fig. 3).

\section{Discussion}

This study demonstrated that a single dose of dezocine $0.1 \mathrm{mg} / \mathrm{kg}$ reduced the incidence and severity of postoperative EA in preschool children undergoing laparoscopic repair of an inguinal hernia by high ligation of the hernia sac under sevoflurane-remifentanil anesthesia. Furthermore, dezocine reduced the use of fentanyl and

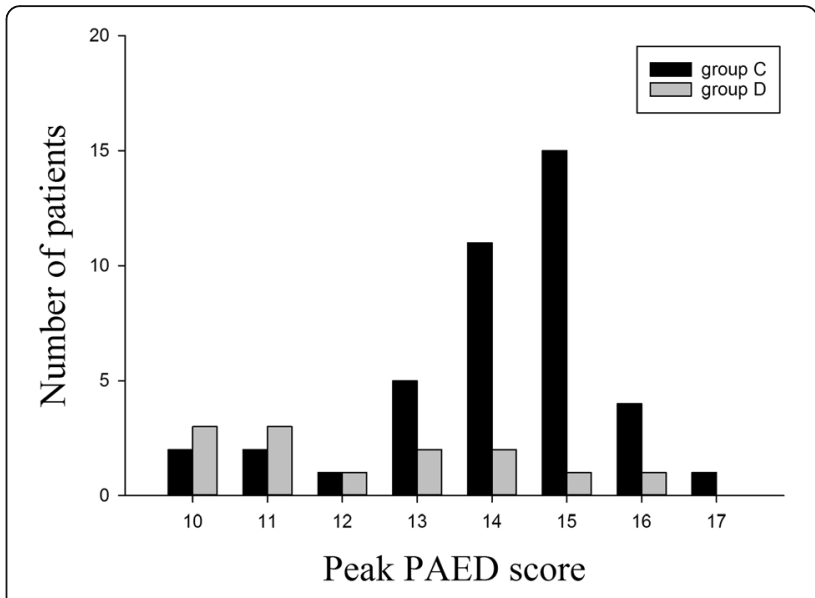

Fig. 2 Distribution of PAED scores

propofol rescue therapy during EA. Although there was a small increase in emergence time in the patients treated with dezocine, there was no delay in discharge from the PACU and no significant differences in the incidence of postoperative oxygen desaturation, headache, nausea, and vomiting between the dezocine and control groups, which suggests that dezocine is not associated with any adverse events in the patient population analyzed.

In our study, dezocine reduced the incidence of EA to $10 \%$ compared to $76 \%$ in the control group. These data are in accordance with a previous study comparing the effects of dezocine, fentanyl, or placebo on the incidence and severity of EA in children undergoing adenotonsillectomy under sevoflurane anesthesia. Findings showed that the incidence (PAED score $\geq 10$ ) and severity of EA

Table $\mathbf{2}$ Emergence agitation and recovery profiles

\begin{tabular}{|c|c|c|c|}
\hline & Group C $(n=49)$ & Group D $(n=49)$ & p-value \\
\hline Time to extubation (min) & $8.1 \pm 1.6$ & $8.6 \pm 1.4$ & 0.103 \\
\hline Time from extubation to emergence (min) & $13.5 \pm 2.1$ & $14.1 \pm 1.7$ & 0.123 \\
\hline Discharged from PACU (min) & $46.4 \pm 8.7$ & $36.5 \pm 9.3$ & 4.032 \\
\hline Incidence of EA by PAED scale score $\geq 10(\%)$ & $42(86 \%)$ & $13(27 \%)$ & $<0.0001$ \\
\hline PAED score $\geq 10$ (moderate EA) & $5(12 \%)$ & $7(54 \%)$ & $<0.0001$ \\
\hline PAED score $\geq 13$ (severe EA) & $37(88 \%)$ & $6(45 \%)$ & $<0.0001$ \\
\hline CHIPPS pain score & $5.2 \pm 0.6$ & $1.2 \pm 0.5$ & $<0.0001$ \\
\hline EA rescued by fentanyl & $9(18 \%)$ & $2(4 \%)$ & 0.025 \\
\hline EA rescued by propofol & $10(20 \%)$ & $0(0 \%)$ & 0.001 \\
\hline Ramsay sedation score & $1.2 \pm 0.5$ & $1.9 \pm 0.4$ & 1.533 \\
\hline Desaturation & 0 & 0 & \\
\hline Nausea & 0 & 0 & \\
\hline Vomiting & 0 & 0 & \\
\hline Headache & 0 & 0 & \\
\hline
\end{tabular}




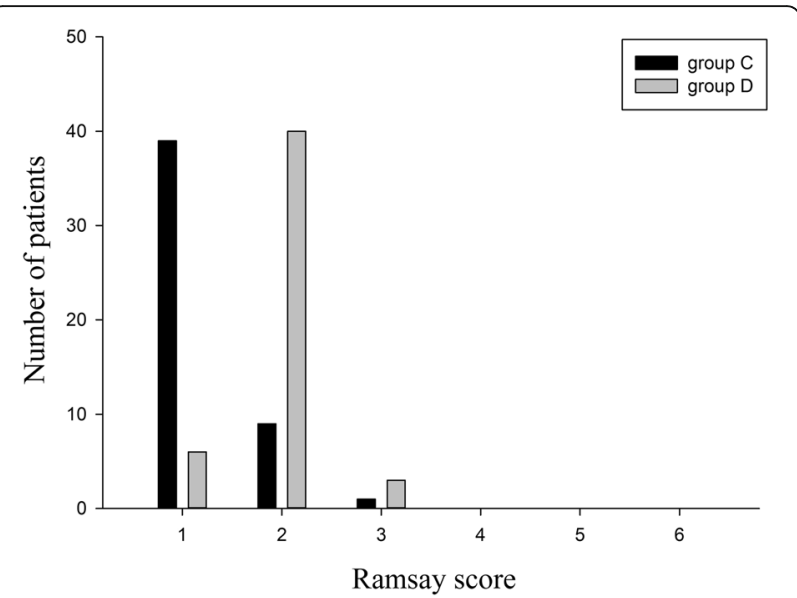

Fig. 3 Ramsey sedation scores for individual patients. The registration information can be found on the following website: http://www.chictr.org.cn/searchproj.aspx

(Severe: Aono's score $\geq 3$ ) was comparable following a single dose of dezocine $0.1 \mathrm{mg} / \mathrm{kg}$ (incidence, $32 \%$; severe $24 \%$ ) and fentanyl $1 \mu \mathrm{g} / \mathrm{kg}$ (incidence, 34\%; severe $26 \%$ ), but lower than placebo (incidence, 61\%; severe 50\%) [18].

The current study used the PAED scale to assess the severity of EA. The PAED scale is a valid and reliable measure of EA in children; however, there is no consensus on the threshold value that indicates the presence of EA. Previous studies have used PAED threshold values ranging from 10 to 16 to identify EA [19-21]. Bajwa et al. reported that a PAED score $>12$ provides greater sensitivity and specificity than a PAED score $\geq 10$; therefore, we defined moderate $\mathrm{EA}$ as $\geq 10$ and $<13$, and severe EA as $\geq 13$ [22].

In the current study, dezocine reduced the use of fentanyl and propofol rescue therapy during EA. Many factors contribute to EA, including patient age, type of surgery, anesthetic procedure, and rapid emergence after surgery. Importantly, pain has been identified as a significant factor in EA. The analgesic effect of dezocine was reflected by a significantly lower CHIPPS score in patients in Group D compared to Group C. Previous studies showed that fentanyl effectively prevents EA in children following sevoflurane anesthesia [23]. However fentanyl also results in chest-wall rigidity and respiratory depression. In the current study, patients in both groups with severe EA were administered intravenous fentanyl. Significantly more patients in Group C required fentanyl rescue compared to Group D, which suggests that dezocine may enhance the analgesic effect of fentanyl. In the patients in our study, pain was mainly caused by surgical incision, stimulation of the gut, and inflammation. Incisional pain was blocked by ropivacaine. Visceralgia was relieved by the $\mathrm{k}$-receptor agonist effect of dezocine in Group D.

The Ramsay sedation score is the most widely used clinical measure of sedation. In this study, patients were evaluated upon admission to the hospital by two neurologists who were not involved in the treatment procedure. The Ramsay sedation score was within the normal range for each child. Postoperatively, there was no significant difference in Ramsay sedation scores between Group C and Group D; however, the number of patients with different levels of sedation varied between groups. A higher number of patients in Group $C$ were evaluated as anxious, agitated or restless compared to Group D, while a greater number of patients in Group D were evaluated as cooperative, orientated, and tranquil or responsive to commands only, compared to Group C. These data indicate that dezocine may reduce the severity of EA though a sedative effect. Evidence suggests that the analgesic and sedative effect of dezocine may be due to inhibition of norepinephrine and serotonin reuptake by their respective transporter proteins. Indeed, in a rat model of chronic constriction injury, dezocine inhibited norepinephrine and serotonin reuptake in a dose dependent manner [24].

This study was associated with several limitations. Firstly, it was a single center study. Multicenter studies are required to verify the safety and efficacy of dezocine for EA in pediatric patients. Secondly, distinguishing between postoperative pain and EA is challenging. Our data may have been confounded by the analgesic effect of dezocine on postoperative pain; indeed, mean CHIPPS score was significantly lower in Group D compared to Group C. Lastly, there was no significant difference in mean Ramsey sedation score between groups, which was unexpected, as dezocine is thought to have sedative effects. Further studies with larger sample sizes are required to verify our findings and elucidate the mechanism of action of dezocine on EA.

\section{Conclusions}

In conclusion, postoperative administration of dezocine $0.1 \mathrm{mg} / \mathrm{kg}$ decreased the incidence and severity of EA in the PACU in preschool children that had undergone laparoscopic repair of an inguinal hernia by high ligation of the hernia sac under sevoflurane-remifentanil anesthesia.

\footnotetext{
Abbreviations

ASA: American Society of Anesthesiologists; BIS: bispectral index; BMI: body mass index; BP: non-invasive blood pressure; CHIPPS: Children and Infants Postoperative Pain Scale; Cl: confidence interval; EA: emergence agitation; HR: heart rate; MAC: minimum alveolar concentration; PACU: post-anesthesia care unit; PAED: Pediatric Anesthesia Emergence Delirium; RRs: risk ratios
} 


\section{Funding}

None.

\section{Availability of data and materials}

The datasets generated and analyzed during the current study are available from the corresponding author on reasonable request.

\section{Authors' contributions}

LJA and STL designed the study. LJA, YZ, ZS and XLZ collected and analyzed the data. LJA, YZ, ZS, HLL, ZJZ and JLH contributed samples collection and intellectual input. LJA and $Y Z$ drafted and wrote the manuscript. LJA and STL revised the manuscript critically for intellectual content. All authors gave intellectual input to the study and approved the final version of the manuscript.

\section{Ethics approval and consent to participate}

This study was approved by the Ethics Committee of Huai'an First People's Hospital, affiliated with Nanjing Medical University (Approval \# HA141001S). All procedures performed in studies involving human participants were in accordance with the ethical standards of the institutional and national research committee and with the 1964 Helsinki declaration and its later amendments or comparable ethical standards. Written informed consent was obtained from the patients' parents or legal guardians.

\section{Consent for publication}

All data published here are under the consent for publication. The consent to publish has been obtained from the participant to report individual patient data.

\section{Competing interests}

The authors declare that they have no competing interests.

\section{Publisher's Note}

Springer Nature remains neutral with regard to jurisdictional claims in published maps and institutional affiliations.

\section{Author details}

'Department of Anesthesiology, Shanghai General Hospital of Nanjing Medical University, University, No. 100 Haining Road, Shanghai 200030, China. ${ }^{2}$ Department of Anesthesiology, Huai'an First People's Hospital of Nanjing Medical University, Huai'an, Jiangsu, China.

Received: 2 April 2017 Accepted: 12 November 2017

Published online: 22 November 2017

\section{References}

1. Aono JUW, Mamiya K, Takimoto E, Manabe M. Greater incidence of delirium during recovery from sevoflurane anesthesia in preschool boys.Pdf. Anesthesiology. 1997:87:1298-300.

2. Cole JWMD, MCAllister JD, Hirshberg GE. Emergence behavior in children_ defining the incidence of excitement and agitation following anaesthesia. Pdf. Paediatr Anaesth. 2002:12:442-7.

3. Na HS, Song IA, Hwang JW, Do SH, Emergence OAY. Agitation in children undergoing adenotonsillectomy: a comparison of sevoflurane vs. sevofluraneremifentanil administration. Acta Anaesthesiol Scand. 2013;57:100-5.

4. Voepel-Lewis T, Malviya S, Tait ARA. Prospective cohort study of emergence agitation in the pediatric Postanesthesia care unit. Anesth Analg. 2003:1625-30.

5. Park JH, Lim BG, Kim HZ, Kong MH, Lim SH, Kim NS, et al. Comparison of emergence agitation between sevoflurane/nitrous oxide administration and sevoflurane administration alone in children undergoing adenotonsillectomy with preemptive ketorolac. Korean J Anesthesiol. 2014;66:34-8.

6. Tan Y, Shi Y, Ding H, Kong X, Zhou H, Tian J. Mu-opioid agonists for preventing emergence agitation under sevoflurane anesthesia in children: a meta-analysis of randomized controlled trials. Paediatr Anaesth. 2016;26:139-50.

7. Kim MS, Moon BE, Kim H, Lee JR. Comparison of propofol and fentanyl administered at the end of anaesthesia for prevention of emergence agitation after sevoflurane anaesthesia in children. Br J Anaesth. 2013;110:274-80.

8. Breschan C, Platzer M, Jost R, Stettner H, Likar R. Midazolam does not reduce emergence delirium after sevoflurane anesthesia in children. Paediatr Anaesth. 2007;17:347-52
9. Hauber JA, Davis PJ, Bendel LP, Martyn SV, McCarthy DL, Evans MC, et al. Dexmedetomidine as a rapid bolus for treatment and prophylactic prevention of emergence agitation in anesthetized children. Anesth Analg 2015;121:1308-15.

10. Park JHLB, Kim H, Lee IO, Kong MH, Kim NS. Comparison of surgical Pleth index-guided analgesia with conventional analgesia practices in Children_a randomized controlled Trial..Pdf. Anesthesiology. 2015;122:1280-7.

11. Curie P. Understanding And Treating Emergence Delirium.pdf. Nurse Anesthesia Capstones. 2015.

12. O'Brien JJBP. Dezocine. A Preliminary review of its pharmacodynamic and pharmacokinetic properties, and therapeutic efficacy.Pdf. Drugs. 1989:38:226-48.

13. Ho RC, Niti M, Kua EH, Ng TP. Body mass index, waist circumference, waisthip ratio and depressive symptoms in Chinese elderly: a population-based study. Int J Geriatr Psychiatry. 2008;23:401-8.

14. Wade TW, Oberhelman SS, Angstman KB, Sawchuk CN, Meunier MR, Angstman GL, et al. Diabetes and obesity not associated with 6-month remission rates for primary care patients with depression. Psychosomatics. 2015;56:354-61.

15. Buttner WFW. Analysis of behavioural and physiological parameters for the assessment of postoperative analgesic demand in newborns, infants and young children_a comprehensive report on seven consecutive studies.Pdf. Paediatr Anaesth. 2000;10:303-18.

16. JA A. The post-anesthesia recovery score revisited.Pdf. J Clin Anesth. 1995;7: 89-91.

17. Wang H, Liu G, Fu W, Li ST. The effect of infraorbital nerve block on emergence agitation in children undergoing cleft lip surgery under general anesthesia with sevoflurane. Paediatr Anaesth. 2015;25:906-10.

18. Li X, Xia Q, Li W. Comparison of the effects of dezocine, fentanyl, and placebo on emergence agitation after sevoflurane anesthesia in children. Int J Clin Pharmacol Ther. 2015:53:241-6.

19. Aouad MT, Y-KVNV, MF El-K, Kanazi GE, Bleik JH. A single dose of propofol at the end of surgery for the prevention of emergence agitation in children undergoing strabismus surgery during sevoflurane anesthesia.Pdf. Anesthesiology. 2007;107:733-8.

20. Abu-Shahwan I, Chowdary K. Ketamine is effective in decreasing the incidence of emergence agitation in children undergoing dental repair under sevoflurane general anesthesia. Paediatr Anaesth. 2007:17:846-50.

21. Abu-Shahwan I. Effect of propofol on emergence behavior in children after sevoflurane general anesthesia. Paediatr Anaesth. 2008;18:55-9.

22. Bajwa SA, Costi D, Cyna AMA. Comparison of emergence delirium scales following general anesthesia in children. Paediatr Anaesth. 2010;20:704-11.

23. Shi F, Xiao Y, Wei X, Zhou Q, Yang P, Huang X. Effects of fentanyl on emergence agitation in children under sevoflurane anesthesia: meta-analysis of randomized controlled trials. PLoS One 2015:10:e0135244.

24. PR WFX, WF Y, Liu R. The anti-nociception effect of Dezocine in a rat neuropathic pain model.Pdf. Transl Perioper. Pain Med. 2014;1:5-8.

\section{Submit your next manuscript to BioMed Central and we will help you at every step:}

- We accept pre-submission inquiries

- Our selector tool helps you to find the most relevant journal

- We provide round the clock customer support

- Convenient online submission

- Thorough peer review

- Inclusion in PubMed and all major indexing services

- Maximum visibility for your research

Submit your manuscript at www.biomedcentral.com/submit
Biomed Central 